\title{
Two bounds for the X-ray transform
}

\section{Richard Oberlin}

Received: 12 October 2008 / Accepted: 8 July 2009 / Published online: 11 August 2009

(C) The Author(s) 2009. This article is published with open access at Springerlink.com

\begin{abstract}
We use the arithmetic-combinatorial method of Katz and Tao to give mixed-norm estimates for the $X$-ray transform on $\mathbb{R}^{d}$ when $d \geq 4$.
\end{abstract}

Keywords $\operatorname{Radon} \cdot \mathrm{X}$-ray $\cdot$ Kakeya

Mathematics Subject Classification (2000) 42B25

\section{Introduction}

Given a function $f$ on $\mathbb{R}^{d}$ and a line $l$ in $\mathbb{R}^{d}$, the $X$-ray transform of $f$ at $l$ is defined

$$
X[f](l)=\int_{l} f(y) d y .
$$

We are interested in estimates for the "Kakeya-order" mixed norm

$$
\|X[f]\|_{L^{q}\left(L^{r}\right)}=\left(\int_{\mathbb{S}^{d-1}}\left(\int_{\xi^{\perp}}|X[f](l(\xi, x))|^{r} d x\right)^{\frac{q}{r}} d \xi\right)^{\frac{1}{q}}
$$

where we integrate with respect to surface measure on the sphere $\mathbb{S}^{d-1}$, where $\xi^{\perp}$ is the orthogonal complement of $\xi$, and where $l(\xi, x)$ is the line parallel to $\xi$ which contains $x$. Specifically, one would like to know which exponents $(p, q, r) \in[1, \infty]^{3}$ allow the bound

$$
\|X[f]\|_{L^{q}\left(L^{r}\right)} \leq C_{p, q, r, d}\|f\|_{L^{p}\left(\mathbb{R}^{d}\right)} .
$$

R. Oberlin $(\bowtie)$

UCLA Mathematics Department, Box 951555, Los Angeles, CA 90095, USA

e-mail: oberlin@math.ucla.edu 
By testing $X$ on characteristic functions of neighborhoods of points and line segments, one sees that

$$
1+\frac{d-1}{r}=\frac{d}{p}
$$

and

$$
\frac{1}{q}+\frac{1}{r} \geq \frac{1}{p}
$$

are necessary conditions for (2) to hold. It is conjectured that, together with the condition $r<\infty$, these are also sufficient. This was shown to be the case for $p<\frac{d+1}{2}$ by Drury in [5] and for $p=\frac{d+1}{2}$ by Christ in [4].

In order to allow for estimates which do not satisfy the endpoint (3), we henceforth only consider the local version of the problem. In this formulation, we impose the additional assumption that $f$ is supported on a fixed ball; the constant in (2) may depend on the ball. The condition (3) then becomes

$$
1+\frac{d-1}{r} \geq \frac{d}{p}
$$

Bounds (2) are closely related to estimates for the Kakeya maximal operator. For $\delta>0$, $\xi \in \mathbb{S}^{d-1}$, and $a \in \mathbb{R}^{d}$, let $l_{\delta}(\xi, a)$ denote the $\delta$-neighborhood of the line segment with endpoints $a, a+\xi$. The Kakeya maximal operator is defined

$$
K[f](\xi)=\sup _{a \in \mathbb{R}^{d}} \frac{1}{\left|l_{\delta}(\xi, a)\right|} \int_{l_{\delta}(\xi, a)}|f(y)| d y .
$$

Above and later, we will use $|\cdot|$ to denote Lebesgue measure, absolute value, or cardinality depending on context. Through an application of Hölder's inequality to the inner norm, one sees that the bound (2) implies the bound

$$
\|K[f]\|_{L^{q}\left(\mathbb{S}^{d-1}\right)} \leq C_{p, q, r, d}^{\prime} \delta^{-\frac{d-1}{r}}\|f\|_{L^{p}\left(\mathbb{R}^{d}\right)} .
$$

This implication does not seem to be reversible. However, it is reasonable to expect that strategies employed for proving (6) may extend to give (2). For example, in [14] Wolff showed that (6) holds with $(p, q, r)=\left(p_{w}, q_{w}, r_{w}-\epsilon\right)$, where

$$
p_{w}=\frac{d+2}{2}, \quad q_{w}=\frac{(d-1)(d+2)}{d}, \quad r_{w}=\frac{(d-1)(d+2)}{d-2},
$$

and later, in [15], he refined the technique to show that when $d=3$ and $f$ is supported on a fixed ball

$$
\|X[f]\|_{L^{q_{w}}\left(L^{r_{w}}\right)} \leq C_{d, \epsilon}\|f\|_{p_{w}, \epsilon}
$$

where $\|\cdot\|_{p, \epsilon}$ denotes the inhomogeneous Sobolev norm with $\epsilon$ derivatives in $L^{p}$. In [9], Łaba and Tao extended this result to higher dimensions, obtaining for $d \geq 4$

$$
\|X[f]\|_{L^{q_{w}}\left(L^{r}\right)} \leq C_{d, \epsilon}\|f\|_{p_{w}, \alpha}
$$

when $r=2(d+2), \alpha=\frac{d-3}{2(d+2)}+\epsilon$.

Katz and Tao [8] have since used arithmetic-combinatorial methods to give estimates for the Kakeya maximal operator which, for $d>8$, are stronger than Wolff's bound (7). They showed that (6) holds with $p=\frac{4 d+3}{7}, q=\frac{4 d+3}{4}$, and $r=\frac{4 d+3}{3}-\epsilon$. We answer the question of whether this may be extended to a result of type (2) affirmatively. 
Theorem 1 When $d \geq 6$ and $\epsilon>0$, the bound (2) holds for all $f$ supported on a fixed ball with $p=\frac{4 d+3}{7}, q=\frac{\overline{4 d}+3}{4}-\epsilon$, and $r=\frac{4 d+3}{3}-\epsilon$.

Except for the $\epsilon$-loss, Theorem 1 is optimal for the ratio $\frac{r}{p}=\frac{7}{3}$ in the sense that it attains the smallest value of $p$ and the largest value of $q$ permitted by (5) and (4). In contrast to [15] and [9], only a minimal refinement of the argument in [8] is needed to obtain Theorem 1.

A subset of $\mathbb{R}^{d}$ is said to be a Kakeya set if it contains a unit line segment in every direction. Bourgain showed in [1] that the bound (6) implies that Kakeya sets must have Hausdorff dimension at least $d-(d-1) \frac{p}{r}$. In [8], Katz and Tao showed that the Hausdorff dimension of a Kakeya set must be at least $(2-\sqrt{2})(d-4)+3$, which is stronger than the result implied by their maximal operator bound. To obtain this dimension estimate, they combined the main estimate from their maximal operator bound with 2-dimensional Kakeya methods, as in the "hairbrush" construction of [14], and used an iterative technique to improve the resulting estimate. We iterate the main estimate from Theorem 1 to obtain

Theorem 2 For $d \geq 4$ and $\epsilon>0$, there exist $p_{\epsilon}, q_{\epsilon}, r_{\epsilon}$ so that (2) holds for all $f$ supported on a fixed ball with $(p, q, r)=\left(p_{\epsilon}, q_{\epsilon}, r_{\epsilon}\right)$ where

$$
\frac{r_{\epsilon}}{p_{\epsilon}}>1+\sqrt{2}-\epsilon, \text { and } \frac{q_{\epsilon}}{p_{\epsilon}}>1+\frac{\sqrt{2}}{2}-\epsilon .
$$

The value of $p_{\epsilon}$ required in Theorem 2 is nonoptimal for the ratio $\frac{r}{p}=1+\sqrt{2}$, and in fact approaches $\infty$ as $\epsilon$ approaches 0 .

The author's interest in mixed-norm estimates (2) was originally motivated by the study of $(d, k)$-Kakeya sets. For $1 \leq k<d, E \subset \mathbb{R}^{d}$ is said to be a $(d, k)$ set if it contains a translate of every $k$-dimensional unit disc. It is conjectured that, for $k>1$, every $(d, k)$ set has positive measure. In [1], Bourgain showed that this is true whenever $2^{k-1}+k \geq d$ (also see previous results $[6,10]$ and related estimates [3]).

Using methods inspired by Bourgain's argument, the following was proven in [11].

Theorem 3 Suppose the bound (2) holds for $f$ supported on a fixed ball. Then $(d, k)$ sets have positive Lebesgue measure whenever

$$
\left(\frac{r}{p}\right)^{k-1}+k>d
$$

From Theorem 2, we thus see that $(d, k)$ sets have positive measure whenever $(1+$ $\sqrt{2})^{k-1}+k>d$. The method from [11] also gives certain estimates for the $k$-plane transform and, in case (8) doesn't hold, lower bounds for the Hausdorff dimension of $(d, k)$ sets.

Theorem 1 is proven in Sects. 2 and 3. The additional arguments needed for Theorem 2 are given in Sect. 4. In an appendix, we show that bounds for the "Nikodym-order" mixed-norms follow from (2).

Remark on notation. We will use $a \lesssim b$ to denote $a \leq C b$ where $C$ is a large constant, $a \ll b$ to denote $a \leq c b$ where $c$ is a small constant, and $a \simeq b$ to denote $a \lesssim b$ and $b \lesssim a$. The size of these constants may on quantities such as $p, q, r, d, \epsilon, N, B$. We will write $1_{E}$ for the characteristic function of a set $E$.

\section{The main estimate}

Let $G \subset \mathbb{R}^{n} \times \mathbb{R}^{n}$. For $t \in \mathbb{R}$ and $(x, y) \in G$, consider the projections into $\mathbb{R}^{n}$

$$
\pi_{t}(x, y)=(1-t) x+t y
$$


and

$$
\pi_{-}(x, y)=y-x .
$$

For our applications, we will identify $G$ with a set of lines in $\mathbb{R}^{n+1}$. This is accomplished by identifying $\mathbb{R}^{n}$ with an $n$-dimensional subspace of $\mathbb{R}^{n+1}$. Then $(x, y)$ will correspond to the the unique line containing the two points $x$ and $e+y$ where $e \in \mathbb{R}^{n+1}$ is orthogonal to $\mathbb{R}^{n}$. For a line $g \in G$, the projection $\pi_{t}(g)$ then gives the intersection of $g$ with the hyperplane $\mathbb{R}^{n}+t e$, and the projection $\pi_{-}(g)$ gives the direction of the line $g$.

We will be seeking upper bounds for the size of $G$ in terms of the sizes of $\pi_{t}(G)$ as $t$ varies over a certain finite set. In [8] (also see the previous works of [2,7]), Katz and Tao worked under the assumption that $G$ was finite, and that the map $\pi_{-}$was injective on $G$. In this setting, the correct notion of "size" is cardinality, and Katz and Tao proved

$$
|G| \leq \sup _{t \in\{0,1,1 / 2,2 / 3\}}\left|\pi_{t}(G)\right|^{7 / 4}
$$

along with several stronger (and more complicated to state) estimates. From these estimates, together with certain discretization and uniformization arguments, they deduced bounds for the Kakeya maximal operator and the dimension of Kakeya sets.

Our aim is to follow Katz and Tao's arguments and account for the possibility that $\pi_{-}$is not injective. For example, if $\pi_{-}$maps at most $M$ points in $G$ to every point in $\mathbb{R}^{n}$, then by the same arguments as in [8], one obtains that

$$
|G| \leq M^{1 / 4} \sup _{t \in\{0,1,1 / 2,2 / 3\}}\left|\pi_{t}(G)\right|^{7 / 4} .
$$

It is worth noting that this is stronger than the bound which follows from (9). The estimates that we will obtain in this manner will allow us to prove mixed-norm estimates for the $X$-ray transform rather than the weaker maximal operator bounds.

The fact that we are not requiring $\pi_{-}$to be injective will also allow us to drop the assumption of finiteness, and instead we will work with sets $G$ of positive Lebesgue measure. Thus, discretization arguments will not be necessary.

We say that a measurable set of lines $G$ is $M$-parallel for $0<M \in \mathbb{R}$ if for every $\xi \in \mathbb{R}^{n}$

$$
\left|\left\{x \in \mathbb{R}^{n}:(x, x+\xi) \in G\right\}\right| \leq M .
$$

In the inequality above, and henceforth, $|\cdot|$ will denote Lebesgue measure rather than cardinality.

The specific bounds we are interested in are of the form

$$
|G| \leq C M^{2-\alpha} \sup _{t \in T}\left|\pi_{t}(G)\right|^{\alpha} .
$$

Above we should have $1 \leq \alpha \leq 2$, and $T$ should be a fixed finite set. The bound should hold for every $M$-parallel set of lines $G$, with $C$ depending only on $n$ and $T$.

Perhaps the most obvious bound of this form is

$$
|G| \leq\left|t_{1}-t_{2}\right|^{-n} \sup _{t=t_{1}, t_{2}}\left|\pi_{t}(G)\right|^{2} .
$$

This is seen to be true by setting

$$
G_{t_{1}, t_{2}}=\left\{\left(\pi_{t_{1}}(g), \pi_{t_{2}}(g)\right): g \in G\right\} .
$$


Then, changing variables,

$$
|G|=\left|t_{1}-t_{2}\right|^{-n}\left|G_{t_{1}, t_{2}}\right|
$$

and clearly $\left|G_{t_{1}, t_{2}}\right| \leq\left|\pi_{t_{1}}(G)\right|\left|\pi_{t_{2}}(G)\right|$.

As a rule of thumb, estimates (10) with a smaller value of $\alpha$ are better (Although important in applications for technical reasons, $C$ and $T$ are secondary considerations). The proposition below is a continuous, non-injective version of the "basic iteration" from [8]. Applying this proposition with the known bound (11) and iterating will allow us to take $\alpha$ arbitrarily close to $1+(\sqrt{2} / 2)$.

Proposition 1 Let $0 \neq s \in \mathbb{R}$ and $u_{1} \neq u_{2} \in \mathbb{R}$. Suppose (10) holds with $T=\left\{t_{1}, \ldots, t_{k}\right\}$ and that $t_{i} \neq u_{j}$ for every $i, j$.

Then, for every $M$-parallel set of lines $G$

$$
|G| \leq C^{\prime} M^{2-\alpha^{\prime}} \sup _{t \in T^{\prime}}\left|\pi_{t}(G)\right|^{\alpha^{\prime}}
$$

where

$$
\begin{aligned}
\alpha^{\prime} & =2-\frac{1}{2 \alpha} \\
T^{\prime} & =\left\{u_{1}, u_{2}, t_{1}, \ldots, t_{k}, t_{1}^{\prime}, \ldots, t_{k}^{\prime}\right\}, \\
t_{i}^{\prime} & =u_{1}+\frac{\left(u_{2}-u_{1}\right)\left(t_{i}-u_{1}\right)}{s\left(t_{i}-u_{2}\right)},
\end{aligned}
$$

and

$$
C^{\prime}=2^{k\left(5 / 2-\alpha^{\prime}\right)} C^{2-\alpha^{\prime}}\left|u_{1}-u_{2}\right|^{-n\left(\alpha^{\prime}-3 / 2\right)} \sup _{i=1, \ldots, k}\left|t_{i}^{\prime}-u_{1}\right|^{-n / 2}
$$

Proof Consider the set

$$
V=\left\{\left(g_{1}, g_{2}\right) \in G^{2}: \pi_{u_{1}}\left(g_{1}\right)=\pi_{u_{1}}\left(g_{2}\right)\right\}
$$

and define projections from $V$ into $G$

$$
\gamma_{i}\left(g_{1}, g_{2}\right)=g_{i}
$$

and from $V$ into $\mathbb{R}^{n}$

$$
v\left(g_{1}, g_{2}\right)=s \pi_{u_{2}}\left(g_{1}\right)+\pi_{u_{2}}\left(g_{2}\right)-\pi_{u_{1}}\left(g_{2}\right) .
$$

The idea of the proof is to find a "nice" point $v_{0} \in v(V)$ and estimate the measure of

$$
G_{v_{0}}=\gamma_{1}\left(\left\{w \in V: v(w)=v_{0}\right\}\right) .
$$

We will find upper and lower bounds for this measure, and combine the bounds to obtain (13).

We start with our upper bound; it will hold for every point $v_{0}$. Continuing to use the notation (12), change variables to obtain

$$
\left|G_{\nu_{0}}\right|=\left|u_{1}-u_{2}\right|^{-n} \int_{\mathbb{R}^{n}} \int_{\mathbb{R}^{n}} 1_{\left(G_{\nu_{0}}\right)_{u_{1}, u_{2}}}(x, y) d x d y .
$$


For $\left(g_{1}, g_{2}\right) \in V$, the direction of the line $g_{2}$ is determined by $\pi_{u_{2}}\left(g_{1}\right)$ and $v$. More specifically, $v\left(g_{1}, g_{2}\right)=\nu_{0}$ is equivalent to $\pi_{u_{2}}\left(g_{2}\right)-\pi_{u_{1}}\left(g_{2}\right)=v_{0}-s \pi_{u_{2}}\left(g_{1}\right)$. Thus the right side of (15) is equal to

$$
\left|u_{1}-u_{2}\right|^{-n} \int 1_{G_{u_{1}, u_{2}}}(x, y) 1_{G_{u_{1}, u_{2}}}\left(x, x+v_{0}-s y\right) d x d y .
$$

Fix $y$ and let $\xi_{y}=\frac{v_{0}-s y}{u_{2}-u_{1}}$. One may calculate that

$$
1_{G_{u_{1}, u_{2}}}\left(x, x+v_{0}-s y\right)=1_{G}\left(x-\xi_{y} u_{1}, x+\xi_{y}\left(1-u_{1}\right)\right)
$$

and so

$$
\int 1_{G_{u_{1}, u_{2}}}\left(x, x+v_{0}-s y\right) d x=\int 1_{G}\left(x, x+\xi_{y}\right) d x \leq M .
$$

The inequality above follows from the hypothesis that $G$ is $M$-parallel. We thus obtain

$$
\left|G_{\nu_{0}}\right| \leq\left|u_{1}-u_{2}\right|^{-n}\left|\pi_{u_{2}}(G)\right| M .
$$

We now proceed to the lower bound for $\left|G_{\nu_{0}}\right|$ which will follow, perhaps counterintuitively, from the hypothesized bound (10).

For any $V^{\prime} \subset V$ and $t, t^{\prime} \neq u_{1}$, let

$$
V_{t, t^{\prime}}^{\prime}=\left\{\left(\pi_{t}\left(g_{1}\right), \pi_{u_{1}}\left(g_{1}\right), \pi_{t^{\prime}}\left(g_{2}\right)\right):\left(g_{1}, g_{2}\right) \in V^{\prime}\right\}
$$

and consider the subset of $V^{\prime}$ which is "popular" with respect to the double projection $\left(\pi_{t} \circ \gamma_{1}, \pi_{t^{\prime}} \circ \gamma_{2}\right)$ :

$\left(V^{\prime}\right)^{t, t^{\prime}}=\left\{\left(g_{1}, g_{2}\right) \in V^{\prime}:\left|\left\{y:\left(\pi_{t}\left(g_{1}\right), y, \pi_{t^{\prime}}\left(g_{2}\right)\right) \in V_{t, t^{\prime}}^{\prime}\right\}\right| \geq \frac{\left|V_{t, t^{\prime}}^{\prime}\right|}{2\left|\pi_{t}(G)\right|\left|\pi_{t^{\prime}}(G)\right|}\right\}$

After estimating $\left|V_{t, t^{\prime}}^{\prime} \backslash\left(V^{\prime}\right)_{t, t^{\prime}}^{t, t^{\prime}}\right|$, one observes that

$$
\left|\left(V^{\prime}\right)_{t, t^{\prime}}^{t, t^{\prime}}\right| \geq \frac{1}{2}\left|V_{t, t^{\prime}}^{\prime}\right|
$$

Consider the refinement of $V$

$$
V^{\prime \prime}=\left(\left(V^{t_{1}, t_{1}^{\prime}}\right)^{t_{2}, t_{2}^{\prime}} \ldots\right)^{t_{k}, t_{k}^{\prime}}
$$

Noting that

$$
\left|V_{t_{i}, t_{i}^{\prime}}^{\prime}\right|\left|\left(t_{i}-u_{1}\right)\left(t_{i}^{\prime}-u_{1}\right)\right|^{-n}=\left|V_{t_{j}, t_{j}^{\prime}}^{\prime}\right|\left|\left(t_{j}-u_{1}\right)\left(t_{j}^{\prime}-u_{1}\right)\right|^{-n}
$$

for every $i, j$ and $V^{\prime} \subset V$, we see from (18) that

$$
\left|V_{t_{i}, t_{i}^{\prime}}^{\prime \prime} \geq 2^{-k}\right| V_{t_{i}, t_{i}^{\prime}} \mid
$$

for every $i$.

Let $G_{\nu_{0}}^{\prime \prime}=\gamma_{1}\left(\left\{w \in V^{\prime \prime}: v(w)=v_{0}\right\}\right)$. Fix $x \in \pi_{t_{i}}\left(G_{v_{0}}^{\prime \prime}\right)$. Then $x=\pi_{t_{i}}\left(g_{1}\right)$ where $\left(g_{1}, g_{2}\right) \in V^{\prime \prime}$ and $v\left(g_{1}, g_{2}\right)=v_{0}$. Letting $z=\pi_{t_{i}^{\prime}}\left(g_{2}\right)$, we have by definition of $V^{\prime \prime}$

$$
\left|\left\{y:(x, y, z) \in V_{t_{i}, t_{i}^{\prime}}\right\}\right| \geq \frac{\left|V_{t_{i}, t_{i}^{\prime}}\right|}{2^{k}\left|\pi_{t_{i}}(G)\right|\left|\pi_{t_{i}^{\prime}}(G)\right|} .
$$


Due to the choice of $t_{i}^{\prime}$ in (14), $v\left(g_{1}, g_{2}\right)$ is determined by $\pi_{t_{i}}\left(g_{1}\right)$ and $\pi_{t_{i}^{\prime}}\left(g_{2}\right)$. Specifically, after some algebra we obtain, for $\left(g_{1}, g_{2}\right) \in V$

$$
v\left(g_{1}, g_{2}\right)=s \frac{u_{2}-u_{1}}{t_{i}-u_{1}} \pi_{t_{i}}\left(g_{1}\right)+\frac{u_{2}-u_{1}}{t_{i}^{\prime}-u_{1}} \pi_{t_{i}^{\prime}}\left(g_{2}\right)
$$

Hence, $y$ satisfies $(x, y, z) \in V_{t_{i}, t_{i}^{\prime}}$ if and only if $(x, y) \in\left(G_{\nu_{0}}\right)_{t_{i}, u_{1}}$. Thus, the left side of (20) is equal to

$$
\left|\left\{y:(x, y) \in\left(G_{v_{0}}\right)_{t_{i}, u_{1}}\right\}\right| .
$$

Since this holds for every $x \in \pi_{t_{i}}\left(G_{v_{0}}^{\prime \prime}\right)$, we obtain

$$
\left|\left(G_{\nu_{0}}\right)_{t_{i}, u_{1}}\right| \geq\left|\pi_{t_{i}}\left(G_{\nu_{0}}^{\prime \prime}\right)\right| \frac{\left|V_{t_{i}, t_{i}^{\prime}}\right|}{2^{k}\left|\pi_{t_{i}}(G)\right|\left|\pi_{t_{i}^{\prime}}(G)\right|}
$$

and it follows that

$$
\left|G_{v_{0}}\right| \geq\left|\pi_{t_{i}}\left(G_{v_{0}}^{\prime \prime}\right)\right| \frac{\left|t_{i}-u_{1}\right|^{-n}\left|V_{t_{i}, t_{i}^{\prime}}\right|}{2^{k}\left|\pi_{t_{i}}(G)\right|\left|\pi_{t_{i}^{\prime}}(G)\right|} .
$$

The set of lines $G_{v_{0}}^{\prime \prime}$ is $M$-parallel by virtue of being contained in $G$. We may thus apply (10) to obtain

$$
\left|G_{\nu_{0}}^{\prime \prime}\right|^{1 / \alpha} \leq C^{1 / \alpha} M^{(2-\alpha) / \alpha} \sup _{i=1, \ldots, k}\left|\pi_{t_{i}}\left(G_{\nu_{0}}^{\prime \prime}\right)\right|
$$

We assume, for the moment, that we may choose $v_{0}$ to be "nice" in the sense that

$$
\left|G_{\nu_{0}}^{\prime \prime}\right| \geq 2^{-k}\left|G_{\nu_{0}}\right|
$$

Combining (22), (23), and (24) gives

$$
1 \leq 2^{k(\alpha+1) / \alpha} C^{1 / \alpha} M^{(2-\alpha) / \alpha}\left|G_{\nu_{0}}\right|^{(\alpha-1) / \alpha} \sup _{i=1, \ldots, k} \frac{\left|\pi_{t_{i}}(G)\right|\left|\pi_{t_{i}^{\prime}}(G)\right|}{\left|t_{i}-u_{1}\right|^{-n}\left|V_{t_{i}, t_{i}^{\prime}}\right|} .
$$

To estimate $\left|V_{t_{i}, t_{i}}\right|$, note

$$
\begin{aligned}
|G| & =\left|t_{i}-u_{1}\right|^{-n} \int 1_{G_{t_{i}, u_{1}}}(x, y) d x d y \\
& \leq\left|t_{i}-u_{1}\right|^{-n}\left|\pi_{u_{1}}(G)\right|^{1 / 2}\left(\int 1_{G_{t_{i}, u_{1}}}(x, y) 1_{G_{t_{i}, u_{1}}}(z, y) d x d y d z\right)^{1 / 2} \\
& =\left|t_{i}-u_{1}\right|^{-n / 2}\left|t_{i}^{\prime}-u_{1}\right|^{-n / 2}\left|\pi_{u_{1}}(G)\right|^{1 / 2}\left|V_{t_{i}, t_{i}^{\prime}}\right|^{1 / 2}
\end{aligned}
$$

where the inequality follows from Cauchy-Schwarz. Combining this with (25) gives

$$
|G|^{2} \leq 2^{k(\alpha+1) / \alpha} C^{1 / \alpha} \sup _{i=1, \ldots, k}\left|t_{i}^{\prime}-u_{1}\right|^{-n} M^{(2-\alpha) / \alpha}\left|G_{\nu_{0}}\right|^{(\alpha-1) / \alpha} \sup _{t \in T^{\prime}}\left|\pi_{t}(G)\right|^{3} .
$$

Combining this with (16) gives (13). 
It remains to find $v_{0}$ satisfying (24). Given any subset $V^{\prime}$ of $V$,

$$
\begin{aligned}
\left|V_{u_{2}, u_{2}}^{\prime}\right| & =\int 1_{V_{u_{2}, u_{2}}^{\prime}}(x, y, z) d x d y d z \\
& =\int 1_{V_{u_{2}, u_{2}}^{\prime}}\left(x, y, v^{\prime}-s x+y\right) d x d y d v^{\prime} \\
& =\int 1_{\left(G_{v^{\prime}}^{\prime}\right)_{u_{1}, u_{2}}}(x, y) d x d y d v^{\prime} . \\
& =\left|u_{1}-u_{2}\right|^{n} \int\left|G_{v^{\prime}}^{\prime}\right| d v^{\prime}
\end{aligned}
$$

where $G_{v^{\prime}}^{\prime}=\gamma_{1}\left\{w \in V^{\prime}: v(w)=v^{\prime}\right\}$. Since $\left|V_{u_{1}, u_{2}}^{\prime \prime}\right| \geq 2^{-k}\left|V_{u_{1}, u_{2}}\right|$, it follows that

$$
\int\left|G_{v^{\prime}}^{\prime \prime}\right| d v^{\prime} \geq \int 2^{-k}\left|G_{v^{\prime}}\right| d v^{\prime}
$$

and so we must have (24) for some $v_{0}$.

\section{Proof of Theorem 1}

We now move on to the task of obtaining mixed-norm estimates for the $X$-ray transform from bounds of the form (10). It will be convenient to reparameterize the $X$-ray transform as follows. Let $e_{1}, \ldots, e_{d}$ be an orthonormal basis for $\mathbb{R}^{d}$, and identify $\mathbb{R}^{d-1}$ with $\operatorname{span}\left(e_{1}, \ldots, e_{d-1}\right)$. For $(\xi, x) \in \mathbb{R}^{d-1} \times \mathbb{R}^{d-1}$ and a function $f$ on $\mathbb{R}^{d}$, we define

$$
T[f](\xi, x)=\int_{0}^{1} f\left(x+t\left(\xi+e_{d}\right)\right) d t
$$

and consider the mixed-norms

$$
\left.\|T[f]\|_{L^{q}\left(L^{r}\right)}=\left(\left.\int_{B}\left(\int_{\mathbb{R}^{d-1}} \mid T[f](\xi, x)\right)\right|^{r} d x\right)^{\frac{q}{r}} d \xi\right)^{\frac{1}{q}}
$$

where $B$ is a fixed ball centered at the origin in $\mathbb{R}^{d-1}$. Through a standard covering argument, one may see that the bound

$$
\|T[f]\|_{L^{q}\left(L^{r}\right)} \lesssim\|f\|_{L^{p}\left(\mathbb{R}^{d}\right)}
$$

is equivalent to the local version of the bound (2).

We will make certain technical reductions in Sects. 3.1 and 3.2 before giving the main uniformization argument in Sect. 3.3.

\subsection{Reduction to weak estimates}

It will be convenient to prove estimates of the form

$$
\lambda M^{1 / r-1 / q}|F|^{1 / q} \lesssim|E|^{1 / p}
$$

where $E$ is contained in the cube $Q=[0,1]^{d}, F \subset B \times \mathbb{R}^{d-1}$, and for every $(\xi, x) \in F$

$$
T\left[1_{E}\right](\xi, x) \geq \lambda
$$


and

$$
\left|\left\{x^{\prime}:\left(\xi, x^{\prime}\right) \in F\right\}\right| \leq M .
$$

We will then obtain estimates (27) with exponents $\left(p_{1}, q_{1}, r_{1}\right)$ arbitrarily close to the exponents $(p, q, r)$ from (28) as follows.

First, since $T$ is local and we will always have $p_{1} \leq q_{1} \leq r_{1}$, it suffices to prove (27) for $f$ supported on the cube $Q$. After a small interpolation with the trivial $L^{1} \rightarrow L^{\infty}\left(L^{1}\right)$ estimate, (27) will follow from the restricted weak type estimate

$$
\left\langle T\left[1_{E}\right], 1_{F}\right\rangle \lesssim|E|^{1 / p_{0}}\left\|1_{F}\right\|_{L^{q_{0}^{\prime}}\left(L^{r_{0}^{\prime}}\right)}
$$

where $E \subset Q, F \subset B \times \mathbb{R}^{d-1},\left(p_{0}, q_{0}, r_{0}\right)$ is arbitrarily close to $(p, q, r)$, and $q_{0}^{\prime}, r_{0}^{\prime}$ are the exponents dual to $q, r$.

Since $E \subset Q$, we may also assume without loss of generality that $F \subset B \times B^{\prime}$ where $B^{\prime} \subset \mathbb{R}^{d-1}$ depends on $B$. In particular, we have for every $(\xi, x) \in F$

$$
\left|\left\{x^{\prime}:\left(\xi, x^{\prime}\right) \in F\right\}\right| \lesssim 1
$$

For integers $i$ with $2^{i} \lesssim 1$ let

$$
F_{i}=\left\{(\xi, x) \in F: 2^{i-1}<\left|\left\{x^{\prime}:\left(\xi, x^{\prime}\right) \in F\right\}\right| \leq 2^{i}\right\}
$$

and for each integer $j$ let

$$
F_{i, j}=\left\{(\xi, x) \in F_{i}: 2^{j-1}<T\left[1_{E}\right](\xi, x) \leq 2^{i}\right\} .
$$

Then

$$
\begin{aligned}
\left\langle T\left[1_{E}\right], 1_{F}\right\rangle & \leq \sum_{2^{i} \lesssim 1} \sum_{j}\left\langle T\left[1_{E}\right], 1_{F_{i, j}}\right\rangle \\
& \lesssim \sum_{2^{i} \lesssim 1} \sum_{j} 2^{j} \min \left(|E|^{q / p} 2^{i(r-q) / r)} 2^{-j q},\left|F_{i}\right|\right) \\
& \leq \sum_{2^{i} \lesssim 1} \sum_{2^{j}<C_{i}} 2^{j}\left|F_{i}\right|+\sum_{2^{i} \lesssim 1} \sum_{2^{j} \geq C_{i}}|E|^{q / p} 2^{i(r-q) / r} 2^{-j(q-1)}
\end{aligned}
$$

where the second inequality follows from (28). Choosing

$$
C_{i}=\frac{|E|^{1 / p} 2^{i(1 / q-1 / r)}}{\left|F_{i}\right|^{1 / q}}
$$

one sees that the right side above is bounded by

$$
\sum_{2^{i} \lesssim 1}\left|F_{i}\right|^{1 / q^{\prime}} 2^{i\left(1 / r^{\prime}-1 / q^{\prime}\right)}|E|^{1 / p} \lesssim|E|^{1 / p}\left\|1_{F}\right\|_{L^{q^{\prime}}\left(L^{r_{0}^{\prime}}\right)}
$$

where $r_{0}$ is any number satisfying $q<r_{0}<r$, and the implicit constant is allowed to depend on $r-r_{0}$. We may thus take $\left(p_{0}, q_{0}\right)=(p, q)$.

\subsection{The two-ends reduction}

In order to obtain almost sharp exponents in Theorem 1, we will need to employ a version of the two-ends reduction from [14]. 
Claim Suppose $q \geq \max \left((d-1) p^{\prime}, p\right)$. Let $\epsilon>0$ be sufficiently small. Suppose (28) holds for all $\lambda$ and all sets $E \subset Q$, and $F \subset B \times \mathbb{R}^{d-1}$, which satisfy

$$
\begin{aligned}
& T\left[1_{E}\right](\xi, x) \geq \lambda, \\
& \left|\left\{x^{\prime}:\left(\xi, x^{\prime}\right) \in F\right\}\right| \leq M .
\end{aligned}
$$

and the additional "two-ends" condition

$$
\left|\left\{t \in I: x+t\left(\xi+e_{d}\right) \in E\right\}\right| \leq 6^{\epsilon}|I|^{\epsilon} T\left[1_{E}\right](\xi, x)
$$

for every $(\xi, x) \in F$ and every interval $I \subset[0,1]$ with $|I| \leq 1 / 6$.

Then, we have

$$
\lambda^{1+3 \epsilon} M^{1 / r-1 / q}|F|^{1 / q} \lesssim|E|^{1 / p}
$$

for all $\lambda, E \subset Q, F \subset \frac{1}{3} B \times \mathbb{R}^{d-1}$ satisfying (29) and (30).

To prove the claim, we will use the following lemma from [13].

Lemma 1 Let $0<\epsilon<1 / 2$. For every $S \subset[0,1]$ there is an interval $J \subset[0,1]$ such that

$$
|J| \geq|S|^{2}
$$

and

$$
|S \cap J| \geq|S|^{1+2 \epsilon}
$$

and

$$
\left|S \cap J^{\prime}\right|<\left(\frac{\left|J^{\prime}\right|}{|J|}\right)^{\epsilon}|S \cap J|
$$

for every interval $J^{\prime}$ with $\left|J^{\prime}\right|<|J|$.

Proof of Lemma 1 Clearly

$$
|S \cap[0,1]| \geq|[0,1]|^{\epsilon}|S| .
$$

Choose an interval $J$ with $|J|$ minimal among all intervals satisfying

$$
|S \cap J| \geq|J|^{\epsilon}|S| .
$$

Since $|S \cap J| \leq|J|$, we have $|J| \geq|S|^{1 /(1-\epsilon)}$. Thus, since $\epsilon<1 / 2$, we obtain (33) and (34). If $\left|J^{\prime}\right|<|J|$, we have by the minimality of $|J|$

$$
\left|S \cap J^{\prime}\right|<\left|J^{\prime}\right|^{\epsilon}|S| \leq\left(\frac{\left|J^{\prime}\right|}{|J|}\right)^{\epsilon}|S \cap J| .
$$

Proof of Claim 3.2 For every $(\xi, x) \in F$ let

$$
S_{\xi, x}=\left|\left\{t: x+t\left(\xi+e_{d}\right) \in E\right\}\right|
$$

and note that $\left|S_{\xi, x}\right|=T\left[1_{E}\right](\xi, x) \geq \lambda$. Apply Lemma 1 with $S=S_{\xi, x}$ to obtain an interval $J_{\xi, x}=J$. For each $(\xi, x)$, we have

$$
\left|J_{\xi, x}\right| \geq|S|_{\xi, x}^{2} \geq \lambda^{2}
$$

and so, after a pigeonhole argument, we may find $R \in\left[\lambda^{2}, 1\right]$ and $F^{\prime} \subset F$ such that $\left|F^{\prime}\right| \gtrsim \lambda^{\epsilon}|F|$ and such that for every $(\xi, x) \in F^{\prime}$ we have $R / 2<\left|J_{\xi, x}\right| \leq R$. 
Let $\mathbf{J}$ be a collection of disjoint intervals of length $R$ covering [0, 1], and for each $J \in \mathbf{J}$ let $3 J$ be the interval with the same center as $J$ but triple the radius. Let

$$
F_{J}=\left\{(\xi, x) \in F^{\prime}: J_{\xi, x} \cap J \neq \varnothing\right\}
$$

and

$$
E_{J}=\left\{\left(x_{1}, \ldots, x_{d}\right) \in E: x_{d} \in 3 J\right\} .
$$

Assume, for the moment, that the inequality

$$
\left|F_{J}\right| \lesssim\left|E_{J}\right|^{q / p} M^{(r-q) / r} \lambda^{-q(1+2 \epsilon)}
$$

holds for every $J \in \mathbf{J}$. Then, since $F^{\prime}=\bigcup_{J \in \mathbf{J}} F_{J}$, we have

$$
|F| \lesssim \lambda^{-\epsilon}\left|F^{\prime}\right| \leq \lambda^{-\epsilon} \sum_{J \in \mathbf{J}}\left|F_{J}\right| \lesssim \lambda^{-\epsilon} \sum_{J \in \mathbf{J}}\left|E_{J}\right|^{q / p} M^{(r-q) / r} \lambda^{-q(1+2 \epsilon)} .
$$

Since the $E_{J}$ 's are finitely overlapping and $q \geq p$, we have

$$
\sum_{J}\left|E_{J}\right|^{q / p} \lesssim|E|^{q / p}
$$

Thus, we conclude

$$
|F| \lesssim|E|^{q / p} M^{(r-q) / r} \lambda^{-(q+\epsilon(1+2 q))}
$$

and hence (32).

It remains to show (35). Note that if $(\xi, x) \in F_{J}$ then, since $\left|J_{\xi, x}\right| \leq|J|$ and $J_{\xi, x} \cap J \neq \emptyset$, we have $J_{\xi, x} \subset 3 J$. Since $\left|S_{\xi, x} \cap J_{\xi, x}\right| \geq\left|S_{\xi, x}\right|^{1+2 \epsilon} \geq \lambda^{1+2 \epsilon}$, we thus have

$$
T\left[1_{E_{J}}\right](\xi, x) \geq \lambda^{1+2 \epsilon}
$$

for every $(\xi, x) \in F_{J}$. Additionally

$$
\left|S_{\xi, x} \cap J^{\prime}\right| \leq\left(\frac{2\left|J^{\prime}\right|}{|J|}\right)^{\epsilon}\left|S_{\xi, x} \cap 3 J\right|
$$

for any interval $J^{\prime}$ with $\left|J^{\prime}\right|<|J| / 2$.

We now rescale to apply the hypothesized bound. Let $t_{0}$ be the center of the interval $J$, and consider the affine map

$$
L\left(x_{1}, \ldots, x_{d}\right)=\left(x_{1}, \ldots, x_{d-1},\left(x_{d}-t_{0}\right) /(3 R)+1 / 2\right) .
$$

Observe that $L\left(E_{J}\right) \subset Q$ and that

$$
\begin{gathered}
x+t\left(\xi+e_{d}\right) \in E_{J} \\
\text { 步 } \\
x+\frac{2 t_{0}-3 R}{2} \xi+\left(\frac{t-t_{0}}{3 R}+\frac{1}{2}\right)\left(3 R \xi+e_{d}\right) \in L\left(E_{J}\right) .
\end{gathered}
$$

We thus set

$$
L^{\prime}\left(F_{J}\right):=\left\{\left(3 R \xi, x+\frac{2 t_{0}-3 R}{2} \xi\right):(\xi, x) \in F_{J}\right\}
$$


and have

$$
T\left[1_{L\left(E_{J}\right)}\right](\xi, x) \geq \lambda^{1+2 \epsilon} /(3 R)
$$

for every $(\xi, x) \in L^{\prime}\left(F_{J}\right)$.

Note that

$$
\left|L\left(E_{J}\right)\right|=\left|E_{J}\right| /(3 R),
$$

that

$$
\left|L^{\prime}\left(F_{J}\right)\right|=(3 R)^{(d-1)}\left|F_{J}\right|,
$$

and that for every $(\xi, x) \in L\left(F_{J}\right)$ we have

$$
\left|\left\{x^{\prime}:\left(\xi, x^{\prime}\right) \in L^{\prime}\left(F_{J}\right)\right\}\right| \leq M .
$$

Finally, from (36) one sees that if $|I| \leq 1 / 6$ then

$$
\left|\left\{t \in I: x+t\left(\xi+e_{d}\right) \in L\left(E_{J}\right)\right\}\right| \leq 6^{\epsilon}|I|^{\epsilon} T\left[1_{L\left(E_{J}\right)}\right](\xi, x)
$$

for every $(\xi, x) \in L^{\prime}\left(F_{J}\right)$.

Applying our hypothesized bound, we obtain

$$
\left|L\left(F_{J}\right)\right| \lesssim\left|L\left(E_{J}\right)\right|^{q / p} M^{(r-q) / r}\left(\lambda^{1+2 \epsilon} /(3 R)\right)^{-q} .
$$

which translates to

$$
\left|F_{J}\right| \lesssim R^{-(d-1)-q / p+q}\left|E_{J}\right|^{q / p} M^{(r-q) / r}\left(\lambda^{1+2 \epsilon}\right)^{-q} .
$$

Provided $q \geq(d-1) p^{\prime}$, we thus obtain (35).

\subsection{Uniformization}

Let $\epsilon>0$ be small, and let $E \subset Q$ and $F \subset B \times \mathbb{R}^{d-1}$ satisfy (29), (30), and (31). We will now prove $(28)$ with $p=(4 d+3) / 7+\tilde{\epsilon}, q=(d-1) p^{\prime}$ and $1 / q-1 / r=1 /(7 p)$ where $\tilde{\epsilon}$ tends to 0 with $\epsilon$. From the reductions in Sects. 3.1 and 3.2, we may thus conclude (27) with $(p, q, r)$ arbitrarily close to $((4 d+3) / 7,(4 d+3) / 4),(4 d+3) / 3)$.

The main estimate we will use, (10), is obtained by applying Proposition 1 to (11).

Corollary 1 Let $s \neq 0$ and $u_{1} \neq u_{2}, t_{1} \neq t_{2}$ satisfy $u_{i} \neq t_{j}$ for each $i, j$. Then for every $M$-parallel set of lines $G \subset \mathbb{R}^{d-1} \times \mathbb{R}^{d-1}$

$$
|G| \leq C M^{1 / 4} \sup _{t=u_{1}, u_{2}, t_{1}, t_{2}, t_{1}^{\prime}, t_{2}^{\prime}}\left|\pi_{t}(G)\right|^{7 / 4}
$$

where

$$
t_{i}^{\prime}=u_{1}+\frac{\left(u_{2}-u_{1}\right)\left(t_{i}-u_{1}\right)}{s\left(t_{i}-u_{2}\right)}
$$

and

$$
C=2^{3 / 2}\left|u_{1}-u_{2}\right|^{-(d-1) / 4}\left|t_{1}-t_{2}\right|^{-(d-1) / 4} \sup _{i=1,2}\left|t_{i}^{\prime}-u_{1}\right|^{-(d-1) / 2}
$$


The following quantity measures the incidences between points in $E$ and lines in $F$

$$
\begin{aligned}
\mathcal{I}(E, F) & =\left\langle 1_{F}, T\left[1_{E}\right]\right\rangle \\
& =\left\langle T^{*}\left[1_{F}\right], 1_{E}\right\rangle \\
& =\int_{\mathbb{R}^{d}} 1_{E}(z) \int_{\mathbb{R}^{d-1}} 1_{F}\left(\xi, z^{\prime}-z_{d} \xi\right) d \xi d z .
\end{aligned}
$$

Above, $z=\left(z_{1}, \ldots, z_{d}\right)$ and $z^{\prime}=\left(z_{1}, \ldots, z_{d-1}\right)$. From (29), we have $\mathcal{I}(E, F) \geq \lambda|F|$.

We break up $E$ into pieces whose $(d-1)$-dimensional slices are of uniform size. Since $E \subset Q$ and $\lambda>0$, we have $F \subset B \times B^{\prime}$ for some ball $B^{\prime}$ depending on $B$. In particular it follows that $|F| \lesssim 1$. Thus, we may assume without loss of generality that $\lambda|F| \leq 1 / 2$ and $\lambda \leq 1 / 2$. Let

$$
S_{0}=\left\{t \in[0,1]:\left|x^{\prime}:\left(x^{\prime}, t\right) \in E\right| \ll \lambda|F|\right\}
$$

and for $k>0$ let

$$
S_{k}=\left\{t \in[0,1]:(\lambda|F|)^{1-(k-1) \epsilon} \lesssim\left|x^{\prime}:\left(x^{\prime}, t\right) \in E\right| \ll(\lambda|F|)^{1-k \epsilon}\right\} .
$$

Since $F \subset B \times \mathbb{R}^{d-1}$, we note that

$$
\int_{\mathbb{R}^{d-1}} 1_{F}\left(\xi, z^{\prime}-z_{d} \xi\right) d \xi \lesssim 1
$$

for every $z$. Hence, defining $E_{k}=\left\{x \in E: x_{d} \in S_{k}\right\}$, we have $\mathcal{I}\left(E_{0}, F\right) \ll \lambda|F| \leq \mathcal{I}(E, F)$. Thus, since $E=\bigcup_{k \lesssim \frac{1}{\epsilon}} E_{k}$, an appropriate choice of implicit constants gives

$$
\mathcal{I}\left(E_{k_{0}}, F\right) \gtrsim \epsilon \mathcal{I}(E, F) \gtrsim \mathcal{I}(E, F) .
$$

for some $k_{0}>0$. Let $E^{\prime}=E_{k_{0}}, S=S_{k_{0}}$, and

$$
F^{\prime}=\left\{(\xi, x) \in F: T\left[1_{E^{\prime}}\right](\xi, x) \gtrsim T\left[1_{E}\right](\xi, x)\right\} .
$$

Considering $\mathcal{I}\left(E^{\prime}, F \backslash F^{\prime}\right)$, we note that

$$
\mathcal{I}\left(E^{\prime}, F^{\prime}\right) \gtrsim \mathcal{I}\left(E^{\prime}, F\right) \gtrsim \lambda|F| .
$$

We will now find a point $\left(u_{1}, u_{2}, t_{1}, t_{1}^{\prime}, t_{2}, t_{2}^{\prime}\right) \in S^{6}$ with which we may apply Corollary 1 to our advantage. Due to the the possible blowup of $C$ in (38), we would like to keep $\left|u_{1}-u_{2}\right|,\left|t_{1}-t_{2}\right|,\left|t_{i}^{\prime}-u_{1}\right|$ suitably large; this will be facilitated by the two-ends condition.

For every $(\xi, x) \in F^{\prime}$, let

$$
S_{\xi, x}=\left\{t^{\prime} \in S: x+t^{\prime}\left(\xi+e_{d}\right) \in E^{\prime}\right\} .
$$

Then, by definition of $F^{\prime}$,

$$
\mu_{\xi, x}:=\left|S_{\xi, x}\right| \gtrsim T\left[1_{E}\right](\xi, x) .
$$

By (31) we have, for every $t \in \mathbb{R}$,

$$
\left|\left\{t^{\prime} \in S_{\xi, x}:\left|t^{\prime}-t\right| \ll 1\right\}\right| \ll T\left[1_{E}\right](\xi, x)
$$

Thus, an appropriate choice of implicit constants gives

$$
\left|\left\{t^{\prime} \in S_{\xi, x}:\left|t^{\prime}-t\right| \gtrsim 1\right\}\right| \gtrsim \mu_{\xi, x}
$$


and so letting

$$
P(\xi, x)=\left\{\left(u_{1}, u_{2}\right) \in S_{\xi, x}^{2}:\left|u_{1}-u_{2}\right| \gtrsim 1\right\},
$$

we have $|P(\xi, x)| \gtrsim \mu_{\xi, x}^{2}$.

For each $\left(u_{1}, u_{2}\right) \in P(\xi, x)$ let

$$
Q_{u_{1}, u_{2}}(\xi, x)=\left\{\left(t_{1}, t_{1}^{\prime}\right) \in S_{\xi, x}^{2}: \min \left(\left|t_{1}-u_{i}\right|,\left|t_{1}^{\prime}-u_{i}\right|\right) \gtrsim 1 \text { for } i=1,2\right\} .
$$

Then, reasoning as above,

$$
\left|Q_{u_{1}, u_{2}}(\xi, x)\right| \gtrsim \mu_{\xi, x}^{2}
$$

for every $\left(u_{1}, u_{2}\right) \in P(\xi, x)$.

Define

$$
s_{u_{1}, u_{2}}\left(t_{i}, t_{i}^{\prime}\right):=\frac{\left(u_{2}-u_{1}\right)\left(t_{i}-u_{1}\right)}{\left(t_{i}-u_{2}\right)\left(t_{i}^{\prime}-u_{1}\right)} .
$$

In order to obtain an $s$ so that (37) holds for each $i$, we consider the set

$$
R_{u_{1}, u_{2}}(\xi, x)=\left\{\left(t_{1}, t_{1}^{\prime}, t_{2}, t_{2}^{\prime}\right) \in Q_{u_{1}, u_{2}}(\xi, x)^{2}: s_{u_{1}, u_{2}}\left(t_{1}, t_{1}^{\prime}\right)=s_{u_{1}, u_{2}}\left(t_{2}, t_{2}^{\prime}\right)\right\} .
$$

Below we abbreviate $s_{u_{1}, u_{2}}$ by $s, Q_{u_{1}, u_{2}}(\xi, x)$ by $Q, R_{u_{1}, u_{2}}(\xi, x)$ by $R$, and $\mu_{\xi, x}$ by $\mu$. We observe that $s\left(t_{1}, \cdot\right)$ is a diffeomorphism on an open set containing the support of $1_{Q}\left(t_{1}, \cdot\right)$, and so we may change variables to obtain

$$
\begin{aligned}
|Q| & =\int_{\mathbb{R}} \int_{\mathbb{R}} 1_{Q}\left(t_{1}, t_{1}^{\prime}\right) d t_{1} d t_{1}^{\prime} \\
& =\int_{\mathbb{R}} \int_{\mathbb{R}} 1_{Q}\left(t_{1}, v_{u_{1}, u_{2}}\left(t_{1}, s^{\prime}\right)\right)\left|\frac{\left(v_{u_{1}, u_{2}}\left(t_{1}, s^{\prime}\right)-u_{1}\right)^{2}\left(t_{1}-u_{2}\right)}{\left(t_{1}-u_{1}\right)\left(u_{2}-u_{1}\right)}\right| d t_{1} d s^{\prime} \\
& \simeq \int_{\mathbb{R}} \int_{\mathbb{R}} 1_{Q}\left(t_{1}, v_{u_{1}, u_{2}}\left(t_{1}, s^{\prime}\right)\right) d t_{1} d s^{\prime}
\end{aligned}
$$

where we define

$$
v_{u_{1}, u_{2}}\left(t_{1}, s\right)=u_{1}+\frac{\left(u_{2}-u_{2}\right)\left(t_{1}-u_{1}\right)}{s\left(t_{1}-u_{2}\right)}
$$

and where the $\simeq$ follows from the fact that the Jacobian is $\simeq 1$ on $Q$. We apply CauchySchwarz and change variables again to see that

$$
\begin{aligned}
& \int_{\mathbb{R}} \int_{\mathbb{R}} 1_{Q}\left(t_{1}, v_{u_{1}, u_{2}}\left(t_{1}, s^{\prime}\right)\right) d t_{1} d s^{\prime} \\
& \leq|s(Q)|^{\frac{1}{2}}\left(\int_{\mathbb{R}} \int_{\mathbb{R}} \int_{\mathbb{R}} 1_{Q}\left(t_{1}, v_{u_{1}, u_{2}}\left(t_{1}, s^{\prime}\right)\right) 1_{Q}\left(t_{2}, v_{u_{1}, u_{2}}\left(t_{2}, s^{\prime}\right)\right) d t_{1} d t_{2} d s^{\prime}\right)^{\frac{1}{2}} \\
& \quad \leq\left(\int_{\mathbb{R}} \int_{\mathbb{R}} \int_{\mathbb{R}} 1_{Q}\left(t_{1}, t_{1}^{\prime}\right) 1_{Q}\left(t_{2}, v_{u_{1}, u_{2}}\left(t_{2}, s\left(t_{1}, t_{1}^{\prime}\right)\right)\right) d t_{1} d t_{1}^{\prime} d t_{2}\right)^{\frac{1}{2}} .
\end{aligned}
$$


Abbreviating

$$
w_{u_{1}, u_{2}}\left(t_{1}, t_{1}^{\prime}, t_{2}\right)=v_{u_{1}, u_{2}}\left(t_{2}, s\left(t_{1}, t_{1}^{\prime}\right)\right)
$$

we have by construction

$$
s\left(t_{1}, t_{1}^{\prime}\right)=s\left(t_{2}, w_{u_{1}, u_{2}}\left(t_{1}, t_{1}^{\prime}, t_{2}\right)\right)
$$

and hence

$$
1_{R}\left(t_{1}, t_{1}^{\prime}, t_{2}, w_{u_{1}, u_{2}}\left(t_{1}, t_{1}^{\prime}, t_{2}\right)\right)=1_{Q}\left(t_{1}, t_{1}^{\prime}\right) 1_{Q}\left(t_{2}, w_{u_{1}, u_{2}}\left(t_{1}, t_{1}^{\prime}, t_{2}\right)\right) .
$$

Thus, we combine (40), (41), and (42), to obtain

$$
|R|:=\int_{\mathbb{R}^{3}} 1_{R}\left(t_{1}, t_{1}^{\prime}, t_{2}, w_{u_{1}, u_{2}}\left(t_{1}, t_{1}^{\prime}, t_{2}\right)\right) d t_{1} d t_{1}^{\prime} d t_{2} \gtrsim \mu^{4} .
$$

In order to control $C$ in (38), it remains to control $\left|t_{1}-t_{2}\right|$. However, since

$$
\int_{\mathbb{R}^{3}} 1_{R}\left(t_{1}, t_{1}^{\prime}, t_{2}, w_{u_{1}, u_{2}}\left(t_{1}, t_{1}^{\prime}, t_{2}\right)\right) 1_{[0, r]}\left(\left|t_{1}-t_{2}\right|\right) d t_{1} d t_{1}^{\prime} d t_{2} \lesssim r \mu^{2},
$$

we have $\left|R^{\prime}\right| \gtrsim \mu^{4}$ where

$$
R^{\prime}=\left\{\left(t_{1}, t_{1}^{\prime}, t_{2}, t_{2}^{\prime}\right) \in R:\left|t_{1}-t_{2}\right| \gtrsim \mu^{2}\right\} .
$$

Let

$$
\begin{aligned}
& X=\left\{\left(\xi, x, u_{1}, u_{2}, t_{1}, t_{1}^{\prime}, t_{2}\right):(\xi, x) \in F^{\prime},\left(u_{1}, u_{2}\right) \in P(\xi, x),\right. \text { and } \\
&\left.\left(t_{1}, t_{1}^{\prime}, t_{2}, w_{u_{1}, u_{2}}\left(t_{1}, t_{1}^{\prime}, t_{2}\right)\right) \in R_{u_{1}, u_{2}}^{\prime}(\xi, x)\right\} .
\end{aligned}
$$

Integrating everything out, we see that

$$
|X| \gtrsim \mathcal{I}\left(E^{\prime}, F^{\prime}\right)\left(\inf _{(\xi, x) \in F^{\prime}} \mu_{\xi, x}\right)^{5} \gtrsim|F| \lambda^{6} .
$$

For each $k \geq 0$ let

$$
X_{k}=\left\{\left(\xi, x, u_{1}, u_{2}, t_{1}, t_{1}^{\prime}, t_{2}\right): \lambda^{2-k \epsilon} \lesssim\left|t_{1}-t_{2}\right| \ll \lambda^{2-(k+1) \epsilon}\right\} .
$$

Then, recalling that each $\mu_{\xi, x} \gtrsim \lambda$ we have by definition of $R_{\xi, x}^{\prime}$

$$
X=\bigcup_{0 \leq k \lesssim \frac{1}{\epsilon}} X_{k}
$$

and hence for some $X^{\prime}:=X_{k_{0}}$, we have $\left|X^{\prime}\right| \gtrsim|X|$. Let $\tilde{\mu}^{2}=\lambda^{2-\left(k_{0}+1\right) \epsilon}$.

Since each $t_{i} \in S$, and each $\left|t_{1}-t_{2}\right| \lesssim \tilde{\mu}^{2}$, we see that the $\left(u_{1}, u_{2}, t_{1}, t_{1}^{\prime}, t_{2}\right)$ reside in a set of measure $\lesssim|S|^{4} \tilde{\mu}^{2}$. Thus we may choose a $\left(u_{1}, u_{2}, t_{1}, t_{1}^{\prime}, t_{2}\right)$ so that, letting

$$
F^{\prime \prime}=\left\{(\xi, x):\left(\xi, x, u_{1}, u_{2}, t_{1}, t_{1}^{\prime}, t_{2}\right) \in X^{\prime}\right\},
$$

we have

$$
\left|F^{\prime \prime}\right| \gtrsim|S|^{-4} \tilde{\mu}^{-2}|F| \lambda^{6}
$$


We now let $G=\left\{(x, x+\xi):(\xi, x) \in F^{\prime \prime}\right\}$ and note that $|G|=\left|F^{\prime \prime}\right|$. Also, since $F^{\prime \prime}$ satisfies $\left|\left\{x^{\prime}:(\xi, x) \in F^{\prime}\right\}\right| \leq M$ for every $(\xi, x) \in F^{\prime \prime}$, we have that $G$ is $M$-parallel. Let $s=s_{u_{1}, u_{2}}\left(t_{1}, t_{1}^{\prime}\right)=s_{u_{1}, u_{2}}\left(t_{2}, t_{2}^{\prime}\right)$. Since $t_{1}^{\prime}, t_{2}^{\prime}$ satisfy (37) we may apply Corollary 1 to obtain

$$
\begin{aligned}
\left|F^{\prime \prime}\right|^{4} \lesssim & \left(\left|u_{1}-u_{2}\right||| t_{1}-t_{2}\left|\min _{i=1,2}\right| t_{i}^{\prime}-\left.u_{1}\right|^{2}\right)^{-(d-1)} M \\
& \cdot \sup _{t=u_{1}, u_{2}, t_{1}, t_{1}^{\prime}, t_{2}, t_{2}^{\prime}}\left|\pi_{t}(G)\right|^{7} \\
\lesssim & \lambda^{-(d-1) \epsilon} \tilde{\mu}^{-2(d-1)} M \sup _{t=u_{1}, u_{2}, t_{1}, t_{1}^{\prime}, t_{2}, t_{2}^{\prime}}\left|\pi_{t}(G)\right|^{7} .
\end{aligned}
$$

By definition of $F^{\prime \prime}, \pi_{t}(G) \subset\left\{x^{\prime}:\left(x^{\prime}, t\right) \in E^{\prime}\right\}$ for each $t$ in the sup above. Thus, we may combine (44) and (45) to obtain

$$
\begin{aligned}
& |F|^{4} \lambda^{24} \tilde{\mu}^{2(d-1)-8} M^{-1} \\
& \quad \lesssim \lambda^{-(d-1) \epsilon}|S|^{16} \sup _{t=u_{1}, u_{2}, t_{1}, t_{1}^{\prime}, t_{2}, t_{2}^{\prime}}\left\{\left.\left(x^{\prime}:\left(x^{\prime}, t\right) \in E^{\prime}\right\}\right|^{7}\right. \\
& \quad \lesssim|E|^{7}|F|^{-7 \epsilon} \lambda^{-(d+6) \epsilon} .
\end{aligned}
$$

Since $\tilde{\mu} \gtrsim \lambda$, we thus have

$$
|F|^{\frac{4}{7}+\epsilon} \lambda^{\frac{14+2 d}{7}+C \epsilon} M^{-\frac{1}{7}} \lesssim|E|
$$

Since $d \geq 6$, we have $4 d+3>14+2 d+C \epsilon$ and hence we obtain the desired estimate.

\section{Proof of Theorem 2}

We will now prove bounds (27) with $r / p$ arbitrarily close to $1+\sqrt{2}$. This improvement over the value $r / p=7 / 3-\epsilon$ from Sect. 2 will be obtained by using an estimate which follows from many successive iterations of Proposition 1 rather than the single iteration which gave us Corollary 1.

Specifically, we start with (11) and apply Proposition 1 iteratively $N-1$ times to obtain Corollary 2 below. Although each iteration decreases the value of $\alpha$ in (10) resulting in an increase of $r / p$ in (27), it will come with the cost of a larger required collection of slices satisfying a more complicated set of arithmetic conditions. This makes the uniformization argument more difficult, and so we are not able to obtain almost sharp values of $p, q, r$.

The heights of the slices will be parameterized by points in $\mathbb{R}^{3 N-1}$ which we will label as

$$
\sigma=\left(u_{1,1}, u_{2,1}, s_{1}, \ldots, u_{1, N-1}, u_{2, N-1}, s_{N-1}, u_{1, N}, u_{2, N}\right)
$$

Provided $u_{1, N} \neq u_{2, N}$, the two-slice estimate (11) holds with $t_{1}=u_{1, N}$ and $t_{2}=u_{2, N}$. Thus, if $u_{1, N-1} \neq u_{2, N-1}, s_{N-1} \neq 0$, and $u_{i, N-1} \neq u_{j, N}$ for $i, j \in\{1,2\}$, then we may apply Proposition 1 with $u_{1}=u_{1, N-1}, u_{2}=u_{2, N-1}$, and $s=s_{N-1}$ to obtain, for every $M$-parallel set of lines $G \subset \mathbb{R}^{n} \times \mathbb{R}^{n}$

$$
|G| \leq C M^{1 / 4} \sup _{t \in \mathcal{T}_{N-1}}\left|\pi_{t}(G)\right|^{\frac{7}{4}}
$$


where

$$
\begin{aligned}
C= & 2^{3 / 2}\left|u_{1, N-1}-u_{2, N-1}\right|^{-n / 4}\left|u_{1, N}-u_{2, N}\right|^{-n / 4} \\
& \cdot \sup _{i=1,2}\left|v_{u_{1, N-1}, u_{2, N-1}}\left(u_{i, N}, s_{N-1}\right)-u_{1, N-1}\right|^{-n / 2}
\end{aligned}
$$

where

$$
\begin{aligned}
\mathcal{T}_{N-1}=\{ & u_{1, N-1}, u_{2, N-1}, u_{1, N}, u_{2, N}, \\
& \left.v_{u_{1, N-1}, u_{2, N-1}}\left(u_{1, N}, s_{N-1}\right), v_{u_{1, N-1}, u_{2, N-1}}\left(u_{2, N}, s_{N-1}\right)\right\}
\end{aligned}
$$

and where

$$
v_{u_{1}, u_{2}}\left(t_{i}, s\right)=u_{1}+\frac{\left(u_{2}-u_{1}\right)\left(t_{i}-u_{1}\right)}{s\left(t_{i}-u_{2}\right)} .
$$

In general, define $\mathcal{T}_{N+1}=\emptyset$, define $s_{N}=1$, and define $\mathcal{T}_{j}$ for $1 \leq j \leq N$ recursively by

$$
\mathcal{T}_{j}=\left\{u_{1, j}, u_{2, j}\right\} \cup \mathcal{T}_{j+1} \cup \mathcal{T}_{j+1}^{\prime}
$$

where $\mathcal{T}_{j+1}^{\prime}=\left\{v_{u_{1, j}, u_{2, j}}\left(t, s_{j}\right): t \in \mathcal{T}_{j+1}\right\}$. We say that $\sigma$ is admissible if for each $1 \leq j \leq$ $N$, we have $u_{1, j} \neq u_{2, j}, s_{j} \neq 0$ and

$$
\left\{u_{1, j}, u_{2, j}\right\} \cap \mathcal{T}_{j+1}=\emptyset .
$$

From $N-1$ iterative applications of Proposition 1, we obtain

Corollary 2 Let $N \geq 2$ and let $\sigma \in \mathbb{R}^{3 N-1}$ be admissible. Then for any $M$-parallel set of lines $G \subset \mathbb{R}^{n} \times \mathbb{R}^{n}$

$$
|G| \leq C_{N}\left(\sup _{t, t^{\prime}}\left|t-t^{\prime}\right|^{-n}\right) M^{2-\alpha_{N}} \sup _{t}\left|\pi_{t}(G)\right|^{\alpha_{N}}
$$

where the right sup ranges over $t \in \mathcal{T}_{1}$, where the left sup ranges over pairs $\left(t, t^{\prime}\right)$ such that

$$
t=u_{1, i}, \quad \text { and } \quad t^{\prime} \in\left\{u_{2, i}\right\} \cup \mathcal{T}_{i+1}^{\prime}, \text { for some } 1 \leq i \leq N
$$

and where

$$
\alpha_{1}=2
$$

and

$$
\alpha_{i+1}=2-\frac{1}{2 \alpha_{i}}
$$

for $1 \leq i<N$.

One may check that $\lim _{i \rightarrow \infty} \alpha_{i}=1+\frac{\sqrt{2}}{2}$. Below, we will use Corollary 2 to show that the estimate (28) holds with $p_{N}, q_{N}, r_{N}$ satisfying

$$
\frac{r_{N}}{p_{N}} \geq \frac{\alpha_{N}}{\alpha_{N}(1+\epsilon)-1}, \quad \text { and } \quad \frac{q_{N}}{p_{N}} \geq \frac{\alpha_{N}}{1+\epsilon \alpha_{N}}
$$

where $\epsilon$ may be taken arbitrarily small. Thus, by taking $N$ large and $\epsilon$ small, we obtain Theorem 2 from the reduction of Sect. 3.1. Since we are not able to obtain anything near a sharp value of $p_{N}$, we will try to keep the arguments as simple as possible rather than pursuing any optimization of this exponent. For example, we will not employ the two-ends reduction. 


\subsection{Counting admissible points}

Motivated by the left sup in (47), we say that $\sigma$ is $c$-admissible if $\left|u_{1, i}-u_{2, i}\right| \geq c$ for every $1 \leq i \leq N$ and $\left|t-t^{\prime}\right| \geq c$ for every $\left(t, t^{\prime}\right)$ such that

$$
t \in\left\{u_{1, i}, u_{2, i}\right\}, \quad \text { and } t^{\prime} \in \mathcal{T}_{i+1} \cup \mathcal{T}_{i+1}^{\prime} \text {, for some } 1 \leq i \leq N .
$$

Proposition 2 Let $I \subset[0,1]$ and let $N$ be a positive integer. Defining

$$
\Omega_{N}(I)=\left\{\sigma \in \mathbb{R}^{3 N-1}: \sigma \text { is } C|I|^{6^{N}}-\text { admissible and } \mathcal{T}_{1} \subset I\right\},
$$

we have

$$
\left|\Omega_{N}(I)\right| \gtrsim|I|^{6^{N}}
$$

Proof The proof will be by induction on $N$. Clearly the proposition is true for $N=1$. We assume it is true for $N=n$, and prove it for $N=n+1$. Let

$$
Q=\left\{\left(u_{1}, u_{2}, t, t^{\prime}\right) \in I^{4}: \min \left(\left|u_{1}-u_{2}\right|,\left|u_{1}-t\right|,\left|u_{1}-t^{\prime}\right|,\left|u_{2}-t\right|,\left|u_{2}-t^{\prime}\right|\right) \gtrsim|I|\right\}
$$

and note that, with an appropriate choice of implicit constant, $|Q| \gtrsim|I|^{4}$.

Define

$$
s_{u_{1}, u_{2}}\left(t, t^{\prime}\right):=\frac{\left(u_{2}-u_{1}\right)\left(t-u_{1}\right)}{\left(t-u_{2}\right)\left(t^{\prime}-u_{1}\right)}
$$

so that

$$
t^{\prime}=v_{u_{1}, u_{2}}\left(t, s_{u_{1}, u_{2}}\left(t, t^{\prime}\right)\right) .
$$

We change variables to obtain

$$
\begin{aligned}
|Q| & =\int 1_{Q}\left(u_{1}, u_{2}, t, v_{u_{1}, u_{2}}(t, s)\right)\left|\frac{\left(v_{u_{1}, u_{2}}(t, s)-u_{1}\right)^{2}\left(t-u_{2}\right)}{\left(t-u_{1}\right)\left(u_{2}-u_{1}\right)}\right| d u_{1} d u_{2} d t d s \\
& \lesssim|I|^{-2} \int 1_{Q}\left(u_{1}, u_{2}, t, v_{u_{1}, u_{2}}(t, s)\right) d u_{1} d u_{2} d t d s .
\end{aligned}
$$

Letting $I_{u_{1}, u_{2}, s}=\left\{t:\left(u_{1}, u_{2}, t, v_{u_{1}, u_{2}}(t, s)\right) \in Q\right\}$ and noting that each $\left(u_{1}, u_{2}, s\right) \in$ $I \times I \times\left[-C|I|^{-2}, C|I|^{-2}\right]$, we then have

$$
\int_{U}\left|I_{u_{1}, u_{2}, s}\right| d u_{1} d u_{2} d s \gtrsim|Q||I|^{2} \gtrsim|I|^{6}
$$

where $U=\left\{\left(u_{1}, u_{2}, s\right):\left|I_{u_{1}, u_{2}, s}\right| \gtrsim|I|^{6}\right\}$. Thus

$$
\int_{U}\left|I_{u_{1}, u_{2}, s}\right|^{6^{n}} d u_{1} d u_{2} d s \gtrsim|I|^{6^{n+1}} .
$$

By the induction hypothesis, we have

$$
\left|\Omega_{n}\left(I_{u_{1}, u_{2}, s}\right)\right| \gtrsim\left|I_{u_{1}, u_{2}, s}\right|^{n^{n}} .
$$

It remains to show that $\left(u_{1}, u_{2}, s, \sigma^{\prime}\right) \in \Omega_{n+1}(I)$ for each $\left(u_{1}, u_{2}, s\right) \in U$ and each $\sigma^{\prime} \in$ $\Omega_{n}\left(I_{u_{1}, u_{2}, s}\right)$. The conclusion (49) will then follow from (51) and (52).

To check that each $\sigma=\left(u_{1}, u_{2}, s, \sigma^{\prime}\right)$ is $C|I|^{6^{n+1}}$-admissible, we first note that $u_{i, j}(\sigma)=$ $u_{i, j-1}\left(\sigma^{\prime}\right)$ and $\mathcal{T}_{j}^{\prime}(\sigma)=\mathcal{T}_{j-1}^{\prime}\left(\sigma^{\prime}\right)$ for $j=2, \ldots, n+1$. Thus, by definition of $\Omega_{n}\left(I_{u_{1}, u_{2}, s}\right)$, 
and the fact that $\left|I_{u_{1}, u_{2}, s}\right| \gtrsim|I|^{6}$ the cases $i=2, \ldots, n+1$ of (48) are covered. To check the case $i=1$, we note that by definition of $\Omega_{n}\left(I_{u_{1}, u_{2}, s}\right)$, we have $\mathcal{T}_{2}(\sigma)=\mathcal{T}_{1}\left(\sigma^{\prime}\right) \subset I_{u_{1}, u_{2}, s}$. By definition of $I_{u_{1}, u_{2}, s}$, we thus have, for every $t \in \mathcal{T}_{2}(\sigma),\left(u_{1}, u_{2}, t, v_{u_{1}, u_{2}}(t, s)\right) \in Q$ and so $\left|u_{1}-u_{2}\right|,\left|u_{1}-t\right|,\left|u_{2}-t\right|,\left|u_{1}-v_{u_{1}, u_{2}}(t, s)\right|,\left|u_{2}-v_{u_{1}, u_{2}}(t, s)\right| \gtrsim|I|$.

Finally, we show that $\mathcal{T}_{1}(\sigma) \subset I$ for each $\sigma=\left(u_{1}, u_{2}, s, \sigma^{\prime}\right)$. Recall

$$
\begin{aligned}
\mathcal{T}_{1}(\sigma) & =\left\{u_{1}, u_{2}\right\} \cup \mathcal{T}_{2}(\sigma) \cup \mathcal{T}_{2}^{\prime}(\sigma) \\
& =\left\{u_{1}, u_{2}\right\} \cup \mathcal{T}_{1}\left(\sigma^{\prime}\right) \cup\left\{v_{u_{1}, u_{2}}(t, s): t \in \mathcal{T}_{1}\left(\sigma^{\prime}\right)\right\} .
\end{aligned}
$$

Since $\sigma^{\prime} \in \Omega_{n}\left(I_{u_{1}, u_{2}, s}\right)$, we have $\mathcal{T}_{1}\left(\sigma^{\prime}\right) \subset I_{u_{1}, u_{2}, s} \subset I$. By definition of $I_{u_{1}, u_{2}, s}$, we also have $\left\{v_{u_{1}, u_{2}}(t, s): t \in \mathcal{T}_{1}\left(\sigma^{\prime}\right)\right\} \subset I$ and $\left\{u_{1}, u_{2}\right\} \subset I$.

\subsection{Uniformization}

Fix $N$ and let $E, E^{\prime}, F, F^{\prime}, M, \lambda, \epsilon, S$, and $S_{\xi, x}$ be as in Sect. 3.3, and

$$
X=\left\{(\xi, x, \sigma):(\xi, x) \in F^{\prime} \text { and } \sigma \in \Omega_{N}\left(S_{\xi, x}\right)\right\} .
$$

Note that, since each $\left|S_{\xi, x}\right| \gtrsim \lambda$, we may apply Proposition 2 to see that

$$
|X| \gtrsim \int_{F^{\prime}}\left|S_{\xi, x}\right|^{6^{N}} d \xi d x \gtrsim \lambda^{6^{N}-1} \mathcal{I}\left(E^{\prime}, F^{\prime}\right) \gtrsim \lambda^{6^{N}}|F|
$$

where each $\sigma$ is $\lambda^{6^{N}}$-admissible and each $\mathcal{T}_{1}(\sigma) \subset S$.

Using the fact that each $\sigma$ is $\lambda^{6^{N}}$-admissible, we obtain the bounds $\lambda^{2 \cdot 6^{N}} \lesssim\left|s_{i}\right| \lesssim \lambda^{-2 \cdot 6^{N}}$ for each $i$ and so, in particular, the $\sigma$ 's reside in a set of measure $|S|^{2 N} \lambda^{-2(N-1) 6^{N}}$. Thus, we may find a fixed $\sigma_{0}$ so that

$$
\left|F^{\prime \prime}\right| \gtrsim \lambda^{(2 N-1) 6^{N}}|S|^{-2 N}|F|
$$

where

$$
F^{\prime \prime}=\left\{(\xi, x):\left(\xi, x, \sigma_{0}\right) \in X\right\} .
$$

Applying Corollary 2 with the set of lines $G=\left\{(x, x+\xi):(\xi, x) \in F^{\prime \prime}\right\}$, we have that $G$ is $M$-parallel and $|G|=\left|F^{\prime \prime}\right|$. Also, since $\mathcal{T}_{1}\left(\sigma_{0}\right) \subset S$, it follows that $\pi_{t}(G) \subset\left\{x^{\prime}:\left(x^{\prime}, t\right) \in\right.$ $\left.E^{\prime}\right\}$ for every $t \in \mathcal{T}_{1}\left(\sigma_{0}\right)$. Finally, since $\sigma_{0}$ is $\lambda^{6^{N}}$-admissible, we have

$$
\sup _{t, t^{\prime}}\left|t-t^{\prime}\right|^{-(d-1)} \lesssim \lambda^{-(d-1) 6^{N}}
$$

in (47). Thus, we obtain

$$
\left|F^{\prime \prime}\right| \lesssim \lambda^{-(d-1) 6^{N}} M^{2-\alpha_{N}} \sup _{t \in S}\left|\left\{x^{\prime}:\left(x^{\prime}, t\right) \in E^{\prime}\right\}\right|^{\alpha_{N}} .
$$

Noting that $\alpha_{N}<2<2 N$ and

$$
|S| \sup _{t \in S}\left|\left\{x^{\prime}:\left(x^{\prime}, t\right) \in E^{\prime}\right\}\right| \lesssim \lambda^{-\epsilon}|F|^{-\epsilon}|E|,
$$

we obtain from (53) and (54)

$$
|F|^{1+\alpha_{N} \epsilon} \lambda^{(2 N+d-2) 6^{N}+\alpha_{N} \epsilon} M^{\alpha_{N}-2} \lesssim|E|^{\alpha_{N}} .
$$


Finally, this gives

$$
\lambda|F|^{\frac{1}{q_{N}}} M^{\left(\frac{1}{r_{N}}-\frac{1}{q_{N}}\right)} \lesssim|E|^{\frac{1}{p_{N}}}
$$

where

$$
\begin{aligned}
p_{N} & =\frac{(2 N+d-2) 6^{N}+\alpha_{N} \epsilon}{\alpha_{N}} \\
\frac{q_{N}}{p_{N}} & =\frac{\alpha_{N}}{1+\alpha_{N} \epsilon} \\
\frac{r_{N}}{p_{N}} & =\frac{\alpha_{N}}{\alpha_{N}(1+\epsilon)-1} .
\end{aligned}
$$

\section{Appendix: Nikodym mixed-norms}

Instead of the Kayeya-order mixed norms (26) for the (reparameterized) $X$-ray transform $T$, one may consider the Nikodym-order mixed norms

$$
\|T[f]\|_{L^{q}\left(L^{r}\right), N}=\left(\int_{\mathbb{R}^{d-1}}\left(\int_{B}|T[f](\xi, x)|^{r} d \xi\right)^{\frac{q}{r}} d x\right)^{\frac{1}{q}} .
$$

In order for the bound

$$
\|T[f]\|_{L^{q}\left(L^{r}\right), N} \lesssim\|f\|_{L^{p}\left(\mathbb{R}^{d}\right)}
$$

to hold, we again have the necessary conditions (4), (5), and $r<\infty$. Unless we impose the additional assumption that $f$ is supported away from the hyperplane $\left\{x: x_{d}=0\right\}$, we have another necessary condition

$$
1+\frac{d-1}{q} \geq \frac{d}{p}
$$

which follows from the application of $T$ to characteristic functions of small neighborhoods of a point in $\left\{x: x_{d}=0\right\}$. Tao showed in [12] that bounds for the Kakeya and Nikodym maximal operators are roughly equivalent. We observe that his proof carries over to the general mixed-norm case, and hence combined with Theorem 1 yields

Corollary 3 When $d \geq 6$ and $\epsilon>0$, the bound (56) holds with $p=\frac{4 d+3}{7}, q=\frac{4 d+3}{4}-\epsilon$, and $r=\frac{4 d+3}{3}-\epsilon$.

One may also formulate a corresponding version of Theorem 2.

Proof For $z \in \mathbb{R}^{d}$ write $z=\left(z^{\prime}, z_{d}\right)$ where $z^{\prime} \in \mathbb{R}^{d-1}, z_{d} \in \mathbb{R}$. For nonnegative integers $j$ let

$$
S_{j}=\left\{z \in \mathbb{R}^{d}: 2^{-(j+1)}<z_{d} \leq 2^{-j}\right\} .
$$

We first prove Corollary 3 in the special case when $f$ is supported on $S_{0}$. In fact, to prove this case it suffices, since $T$ is local and $p \leq q \leq r$, to consider the case when $f$ is supported on $S_{0} \cap Q$ where $Q$ is the cube centered at $\frac{1}{2} e_{d}$ with side length 1 . Furthermore, assume that $f$ is non-negative. 
Consider the projective transformation

$$
\phi(z)=\frac{\left(z^{\prime}, 1\right)}{z_{d}} .
$$

Letting $d_{2}(y)=2 y$, we have

$$
\begin{aligned}
T\left[f \circ \phi \circ d_{2}\right](\xi, x) & =\int_{\frac{1}{2}}^{1} f \circ \phi \circ d_{2}\left(x+t\left(\xi+e_{d}\right)\right) d t \\
& =\int_{\frac{1}{2}}^{1} f\left(\xi+\frac{1}{2 t}\left(2 x+e_{d}\right)\right) d t \\
& \simeq \int_{\frac{1}{2}}^{1} f\left(\xi+\tilde{t}\left(2 x+e_{d}\right)\right) d \tilde{t} \\
& =T[f](2 x, \xi),
\end{aligned}
$$

where the first and last equations follow from the fact that $f$ is supported on $S_{0}$. Thus by Theorem 1

$$
\begin{aligned}
& \left(\int_{\mathbb{R}^{d-1}}\left(\int_{B}|T[f](\xi, x)|^{r} d \xi\right)^{\frac{q}{r}} d x\right)^{\frac{1}{q}} \\
& =\left(\int_{B^{\prime}}\left(\int_{B}|T[f](\xi, x)|^{r} d \xi\right)^{\frac{q}{r}} d x\right)^{\frac{1}{q}} \\
& \lesssim\left(\int_{B^{\prime}}\left(\int_{B} \mid T\left[f \circ \phi \circ d_{2}\right]\left(x, \frac{1}{2} \xi\right)^{r} d \xi\right)^{\frac{q}{r}} d x\right)^{\frac{1}{q}} \\
& \lesssim\left(\int_{B^{\prime}}\left(\int_{\mathbb{R}^{d-1}}\left|T\left[f \circ \phi \circ d_{2}\right](x, \xi)\right|^{r} d \xi\right)^{\frac{q}{r}} d x\right)^{\frac{1}{q}} \\
& \lesssim\left\|f \circ \phi \circ d_{2}\right\|_{L^{p}\left(\mathbb{R}^{d}\right) .}
\end{aligned}
$$

where we use the fact that $f$ is supported on $Q$ for the first equation. Since $f$ is supported on $S_{0}$, we have

$$
\left\|f \circ \phi \circ d_{2}\right\|_{L^{p}\left(\mathbb{R}^{d}\right)} \lesssim\|f\|_{L^{p}\left(\mathbb{R}^{d}\right)} .
$$

Lifting our support assumptions on $f$, we note that

$$
\|T[f]\|_{L^{q}\left(L^{r}\right), N} \leq \sum_{j=0}^{\infty}\left\|T\left[1_{S_{j}} f\right]\right\|_{L^{q}\left(L^{r}\right), N} .
$$


However each $1_{S_{j}} f \circ d_{2^{-j}}$ is supported on $S_{0}$, and

$$
T\left[1_{S_{j}} f\right](\xi, x)=2^{-j} T\left[1_{S_{j}} f \circ d_{2^{-} j}\right]\left(\xi, 2^{j} x\right) .
$$

Thus, for each $j$

$$
\begin{aligned}
\left\|T\left[1_{S_{j}} f\right]\right\|_{L^{q}\left(L^{r}\right), N} & =2^{-j\left(1+\frac{d-1}{q}\right)}\left\|T\left[1_{S_{j}} f \circ d_{2^{-j}}\right]\right\|_{L^{q}\left(L^{r}\right), N} \\
& \lesssim 2^{-j\left(1+\frac{d-1}{q}\right)}\left\|1_{S_{j}} f \circ d_{2^{-j}}\right\|_{L^{p}\left(\mathbb{R}^{d}\right)} \\
& \leq 2^{-j\left(1+\frac{d-1}{q}-\frac{d}{p}\right)}\|f\|_{L^{p}\left(\mathbb{R}^{d}\right)} .
\end{aligned}
$$

Hence, since (57) holds with strict inequality, we have

$$
\sum_{j=0}^{\infty}\left\|T\left[1_{S_{j}} f\right]\right\|_{L^{q}\left(L^{r}\right), N} \lesssim\|f\|_{L^{p}\left(\mathbb{R}^{d}\right)} .
$$

Open Access This article is distributed under the terms of the Creative Commons Attribution Noncommercial License which permits any noncommercial use, distribution, and reproduction in any medium, provided the original author(s) and source are credited.

\section{References}

1. Bourgain, J.: Besicovitch type maximal operators and applications to Fourier analysis. Geom. Funct. Anal. 1(2), 147-187 (1991)

2. Bourgain, J.: On the dimension of Kakeya sets and related maximal inequalities. Geom. Funct. Anal. 9(2), 256-282 (1999)

3. Bueti, J.: An incidence bound for $k$-planes in $F^{n}$ and a planar variant of the Kakeya maximal function. Preprint, arXiv:math.CO/0609337

4. Christ, M.: Estimates for the $k$-plane transform. Indiana Univ. Math. J. 33(6), 891-910 (1984)

5. Drury, S.W.: $L^{p}$ estimates for the X-ray transform. Ill. J. Math. 27(1), 125-129 (1983)

6. Falconer, K.J.: Continuity properties of $k$-plane integrals and Besicovitch sets. Math. Proc. Camb. Philos. Soc. 87(2), 221-226 (1980)

7. Katz, N.H., Tao, T.: Bounds on arithmetic projections, and applications to the Kakeya conjecture. Math. Res. Lett. 6(5-6), 625-630 (1999)

8. Katz, N.H., Tao, T.: New bounds for Kakeya problems. J. Anal. Math. 87, 231-263 (2002). Dedicated to the memory of Thomas H. Wolff

9. Łaba, I., Tao, T.: An X-ray transform estimate in $\mathbb{R}^{n}$. Rev. Mat. Iberoam. 17(2), 375-407 (2001)

10. Marstrand, J.M.: Packing planes in $\mathbf{R}^{3}$. Mathematika 26(2), 180-183 (1979)

11. Oberlin, R.: The $(d, k)$ Kakeya problem, and estimates for the X-ray transform. Ph.D. thesis, University of Wisconsin-Madison (2007)

12. Tao, T.: The Bochner-Riesz conjecture implies the restriction conjecture. Duke Math. J. 96(2), 363-375 (1999)

13. Tao, T., Wright, J.: $L^{p}$ improving bounds for averages along curves. J. Am. Math. Soc. 16(3), 605-638 (2003) (electronic)

14. Wolff, T.: An improved bound for Kakeya type maximal functions. Rev. Mat. Iberoam. 11(3), 651674 (1995)

15. Wolff, T.: A mixed norm estimate for the X-ray transform. Rev. Mat. Iberoam. 14(3), 561-600 (1998) 